\title{
Convective heat transfer from a surface to a slot air jet using infrared thermography: Effect of the temperature on flux measurements
}

\author{
by P.Gervais *, E.Estiot *and D. Varieras * \\ * Centre de Thermique de Lyon, UMR CNRS 5008 \\ Institut National des Sciences Appliquées de Lyon \\ 20, avenue A. Einstein, 69621 Villeurbanne, France
}

\begin{abstract}
Impinging jet heat transfer is investigated experimentally using the steady heatedthin-foil technique associated to infrared thermography. For low Reynolds numbers the convection heat flux in the stagnation region is very sensitive to the target surface temperature measurements. This is mainly due to the tangential heat conduction term in the local balance equation. This term is also responsible of the measurement uncertainty increase.
\end{abstract}

\section{Introduction}

The steady Heated-Thin-Foil technique is widely used for impinging jet heat transfer measurements [1]. This technique consists in heating a thin metallic foil by Joule effect and then to determine the convective heat transfer between the jet and the heated foil by means of a local energy balance.

There are two main techniques for temperature measurements: thermocouples [2] and infrared thermography [3]. Infrared thermography measurements are particularly well adapted to the non-uniform heat transfer involved by impinging jets. However, it generates some difficulties linked to the use of a radiometric equation and also the necessity to manage an optical access to the heated surface.

The present experiments are conducted for a slot air-jet impinging on a flat plate [4]. The impinging surface thickness constraints us to take into account the tangential heat conduction fluxes. The Laplacian calculation lead us to focus a particular attention on temperature measurement uncertainties. Error modelling and uncertainty analysis on each term of the energy balance equation allow to make a measurement optimisation versus the air flow parameters.

\section{Experimental apparatus and procedures}

Figure 1 shows the overall schematic of, the experimental apparatus. The compressed air flow is filtered and depressed ranging [0-1.5] bars by means of a regulator valve. Then the air is passed through a plenum and brought into a rectangular channel, $5 \mathrm{~mm} \times 150 \mathrm{~mm}$ cross section and $200 \mathrm{~mm}$ long, from which output the slot jet is produced. The Reynolds numbers are ranging from 280 to 4300 for this experimental setup. The nozzle-to-plate distance is $20 \mathrm{~mm}$. A stainless steel plate, $100 \mathrm{~mm}$ wide, $300 \mathrm{~mm}$ long $\left(I_{p}\right)$ and $0.15 \mathrm{~mm}$ thick $\left(e_{p}\right)$, is used as the target surface. The electrical resistivity of the steel foil $r(T)$ has been measured, versus temperature. A variable low voltage transformer ( $240 \mathrm{~V}-7 \mathrm{~V})$ has been used to heat the foil and a calibrated resistance $R_{S}$ allows to accurately measure the electrical power dissipated into the foil. The rear face of the heated foil is imaged with an infrared scanning radiometer (Nippon Avionics TVS MK2000). The rear face of the foil is 
coated with a paint which emissivity coefficient has been determined to be uniform in the range of the detector spectral response, i.e. [3-5.5] $\mu \mathrm{m}$. All the emissivity and reflectivity coefficients, that are needed in this experiment, have been measured using spectrometers (BIORAD 60A model for wavelength ranging [1.5-25 ] $\mu \mathrm{m}$ and PERKIN ELMER Lambda 900 for [0.3-2.5] $\mu \mathrm{m}$ ). On the jet side, the front face of the foil has a high reflectivity coefficient $(\rho=0.82)$. Average emissivity or reflectivity coefficients have been calculated from a Planck's law weighted sum in the range of [0.3-25] $\mu \mathrm{m}$. The confinement wall is covered with a high reflective layer $(\rho=0.83)$ in order to reduce the radiative heat transfer term in the power balance equation.

For each image $k$, the temperature $T_{k}(i, j)$ at $(i, j)$ image point, has been derived from the thermal signal $S_{k}(i, j)$ by means of the radiometric equation:

$$
S_{k}(i, j)=\varepsilon V\left(T_{k}(i, j)\right)+(1-\varepsilon) V\left(T_{e n v}\right)
$$

The radiometer to target distance is $400 \mathrm{~mm}$, then atmospheric absorption is not taken into account. $T_{e n v}$ is assumed to be close to the ambient air temperature $T_{a m b}$.

The two-dimensional jet flow allows to only consider the temperature profile along the target surface. Then, temperature profile $T(i)$ is calculated as follows :

$$
T(i)=\frac{1}{100} \sum_{k=1}^{N} \frac{1}{10} \sum_{j=l_{c}}^{l+10} T_{k}(i, j)
$$

Where subscript $j$ stands for the 10 image lines that are scanned around the central detector at line $I_{c}$.

\section{Data-reduction equations}

The local energy balance has been written for each image element (referenced as $i$ ). The Point Spread Function (PSF) of the imager has been identified and no sensible PSF effects have been shown considering the encountered temperature gradients. Consequently, the number of measurement points is assumed to be equal to the number of pixel .

The balance equation in steady state, for an image point $i$ of $\Delta x \Delta y$ area, is :

$$
\Phi_{\text {conv }}(i)=P(i)-\left(\Phi_{r f t}(i)+\Phi_{r a d}(i)+\Phi_{\text {cond }}(i)\right)
$$

Where $P(i)$ is the electrical power supplied at point $i$ :

$$
P(i)=\frac{r(T(i)) V_{s}^{2}}{e_{p} l_{p}^{2} R_{s}^{2}} \Delta x \Delta y
$$

The target surface thickness $e_{p}$ is relatively small to neglect the temperature difference between each side of the target surface, but not too small to neglect the tangential conduction term:

$$
\Phi_{\text {cond }}(i)=-\lambda_{p} e_{p} \Delta x \Delta y\left(\frac{\partial^{2} T(i)}{\partial x^{2}}\right)
$$

The Laplacian calculation is based on the well-known Stavisky and Golay's algorithm. In order to reduce noise magnification the convolution kernel dimension $N$ has been chosen to 25 : 


$$
\frac{\partial^{2} T(i)}{\partial x^{2}}=\sum_{n=i-\frac{N}{2}}^{i+\frac{N}{2}} a_{n} T(i)
$$

The radiative heat losses $\Phi_{r a d}(i)$ have been calculated using the radiosity method. The results point out that, for low emissivity coefficients of internal surfaces, the following simplified formulation does not present a relative difference greater than $2 \%$, compared to the radiosity method:

$$
\Phi_{r a d}(i)=\varepsilon_{p} \sigma \Delta x \Delta y\left(T^{4}(i)-T_{a m b}^{4}\right)
$$

The rear face heat losses $\Phi_{r f l}(i)$ have been accurately calibrated, as a function of the temperature difference between the target surface and the ambient air, using a special experiment. Consequently, a calibration curve (polynomial of order 4) and a measurement uncertainty (table 1) have been defined [5].

\section{Uncertainty analysis}

All following uncertainties are reported at a $95 \%$ confident level. The infrared scanning radiometer has been calibrated, in the range of [300-400] $\mathrm{K}$, on a grey and scattering surface whose normal emissivity coefficient has been determined to be $0.99 \pm 0.01$ in the spectral response interval of the detectors. The thermal signal conversion (Eq.1) takes into account the emissivity coefficient of the surface $\varepsilon$, the environmental temperature $T_{e n v}$ and the digital-to-analog signal delivered by the radiometer $S$. The total temperature uncertainty has been derived from a quadratic combination [6] of the total uncertainty of each of these 3 parameters. Finally, the total temperature uncertainty interval is $\pm[0,4-0,8] \mathrm{K}$ when temperature ranges from 300 to $400 \mathrm{~K}$. The convection heat flux uncertainty is defined as follows :

$$
\Delta \Phi_{\text {conv }}(i)=\sqrt{(\Delta P(i))^{2}+\left(\Delta \Phi_{\text {cond }}(i)\right)^{2}+\left(\Delta \Phi_{r a d}(i)\right)^{2}+\left(\Delta \Phi_{r f l}(i)\right)^{2}}
$$

Uncertainty calculations for each flux terms, $\Delta \Phi_{x}(i)$, have been achieved as a function of all sensitivity coefficients $\partial \Phi_{x}(i) / \partial X^{j}$, where $X_{j}$ stands for a measurement variable, and all total uncertainties $\Delta X_{j}$. Table 1 reports these measurement variables and measurement uncertainties.

\section{Results and discussion}

In the following results, the Reynolds numbers are set at 280, 1360 and 4300 . The electrical current that passes through the stainless steel foil is $30 \mathrm{~A}$. The environmental air temperature is respectively $18.3,21.7$ and $22{ }^{\circ} \mathrm{C}$ for these three tests. The elementary surface area $\Delta x \Delta y$ is $0.563 \times 0.275 \mathrm{~mm}^{2}$. It should be noted that the $x / e$ axis origin belongs to the symmetry plane of the flow. Consequently, result curves are only reported for positive $x / e$ axis.

The rear surface temperature profiles have been reported on figure2. As the Reynolds number increases, the thermal contrast decreases. This is due to a higher efficiency of the cooling process. The thermal efficient zone can be correlated to the first zero location of the temperature second derivative. Figure 3 shows that the first 
zero location is significantly displaced downstream $(x / e=2.2)$ for a low Reynolds number.

Figure 4 shows the convective heat fluxes versus $x / e$ axis. Two cases have been compared:

Case A: The power balance is described by Eq. 2

Case B: The conduction term is not taken into account in the balance equation, i.e. the new balance equation is:

$$
\Phi_{\text {conv }}(i)=P(i)-\left(\Phi_{r f l}(i)+\Phi_{r a d}(i)\right)
$$

Table 2 reports the values of the convective heat flux and associated measurement uncertainties at $\mathrm{x} / \mathrm{e}=0$, for the presented experiments.

Several major remarks are pointed out. First of all, on figure 4, the convective heat flux value in the stagnation zone shows a relative default of $47 \%$ for Case $B$ and for $\operatorname{Re}=280$. This bias is only $18 \%$ if $\operatorname{Re}=1360$ and decays to $6.7 \%$ if $\operatorname{Re}=4300$. This important shift is particularly high in the stagnation zone, it vanishes downstream and the two Cases lead to roughly the same results.

Measurement uncertainties increase slightly for Case A. This effect is due to the high number of temperature measurements needed for the second derivative calculation.

The average heat fluxes along the target plate are shown in a column graph (figure 5). The reference value is the total power supplied into the target surface. It clearly appears that the average conduction and radiation terms are negligible compared to the rear face heat losses and, fortunately, to the convective heat flux. However, these average heat fluxes cannot become a reference criterion in order to simplify the data-reduction equation.

\section{Conclusion}

The convective heat flux from a plane surface to a slot air jet has been measured using the well known steady heated thin foil technique. The target surface temperature measurements have been achieved with an infrared scanning radiometer. The tangential heat conduction term is of importance in the local power balance equation. This major role underlined the temperature measurements quality when low Reynolds numbers are involved. Measurement uncertainties have been examined and show a slight increase if the tangential conduction heat transfer is taken into account in the balance equation.

\section{REFERENCES}

[1] T. Astarita, G. Cardone, G.M. Carlomagno, C. Meola, A survey on infrared thermography for convective heat transfer measurements, Optics and laser technology, 32, pp 593-610, (2000)

[2] M. Rahimi, I. Owen, J. Mistry, Impingement heat transfer in an under-expanded axisymetric air jet, Int. Journal of Heat and Mass Transfer, Vol.46, pp 263-272, (2003)

[3] C. Meola, L. de Luca, G. M. Carlomagno, Influence of shear layer dynamics on impingement heat transfer, Experimental Thermal and Fluid Science, 13, pp 29-37, ( 1996)

[4] Varieras D., Gervais P., Giovannini A., Breton J.L., Laser tomographic and interferometric visualizations applied to a confined laminar slot jet impinging on a flat plate, Journal of Flow Visualization and Image Processing, Vol. 8, Nos. 2\&3, pp 91109, ( 2001)

[5] Neuilly M., Modélisation et estimation des erreurs de mesure, Technique et Documentation, Lavoisier (1993) 
[6] R.J. Moffat, Describing the uncertainties in experimental results, Experimental Thermal and Fluid Science, 1, pp 3-17,( 1988)

Table 1. Measurement variables and uncertainties

\begin{tabular}{|c|c|c|c|}
\hline$X^{j}$ & $\Delta X^{j}$ & $X^{j}$ & $\Delta X^{j}$ or $\Delta X^{j} X^{j}$ \\
\hline$\lambda_{\mathrm{p}}$ & $\pm 0.09 \mathrm{~W} / \mathrm{m} . \mathrm{K}$ & $T_{\text {amb }}, T_{\text {env }}$ & $\pm 0.3 \mathrm{~K}$ \\
\hline$e_{\mathrm{p}}$ & $\pm 5 \mu \mathrm{m}$ & $\Delta x, \Delta y$ & $\pm 1 \%$ \\
\hline$I_{\mathrm{p}}$ & $\pm 13 \mu \mathrm{m}$ & $T_{i}$ & $\pm 0.3 \mathrm{~K}$ \\
\hline$V_{\mathrm{s}}$ & $\pm 1.5 \mu \mathrm{V}$ & $\varepsilon$ & $\pm 1 \%$ \\
\hline$R_{\mathrm{s}}$ & $\pm 1.3 \mu \Omega$ & $S$ & $\pm 0.1 \%$ \\
\hline$r$ & $\pm 0.1 \mu \tilde{\Omega} \mathrm{m}$ & $\Phi_{\text {rfl }}$ & $\pm 0.3 \%$ \\
\hline
\end{tabular}

Table 2. Convective heat flux and uncertainties, at x/e axis origin

\begin{tabular}{|c|c|c|c|c|c|c|}
\cline { 2 - 7 } \multicolumn{1}{c|}{} & \multicolumn{3}{c|}{ With conduction terms $\times 10^{4} \mathrm{~W}$} & \multicolumn{3}{|c|}{ Without conduction terms $\times 10^{4} \mathrm{~W}$} \\
\hline $\operatorname{Re}$ & $\Phi_{\text {conv }}$ & $\Delta \Phi_{\text {conv }}$ & $\Delta \Phi_{\text {conv }} / \Phi_{\text {conv }}$ & $\Phi_{\text {conv }}$ & $\Delta \Phi_{\text {conv }}$ & $\Delta \Phi_{\text {conv }} / \Phi_{\text {conv }}$ \\
\hline 280 & 2.68 & \pm 0.28 & $10 \%$ & 1.82 & \pm 0.25 & $13 \%$ \\
\hline 1360 & 2.67 & \pm 0.27 & $10 \%$ & 2.25 & \pm 0.25 & $11 \%$ \\
\hline 4300 & 2.53 & \pm 0.28 & $11 \%$ & 2.38 & \pm 0.25 & $10 \%$ \\
\hline
\end{tabular}

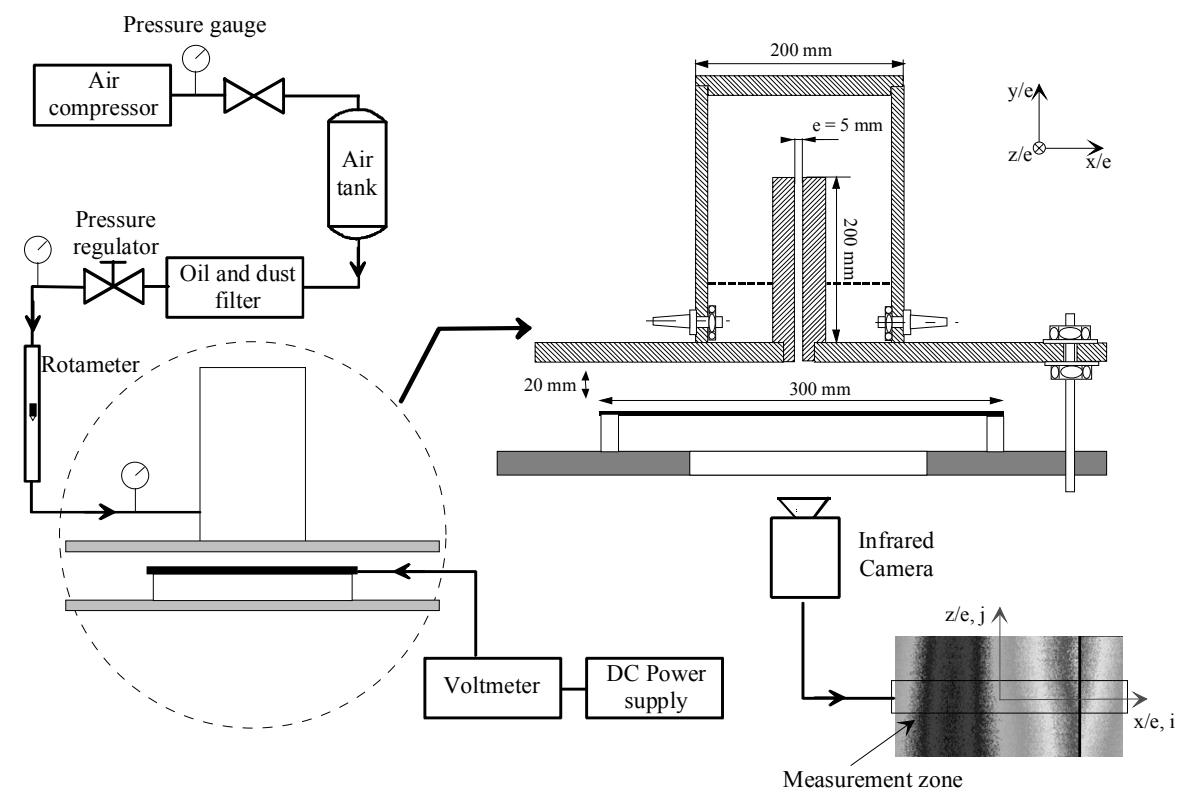

Fig. 1. Experimental apparatus 


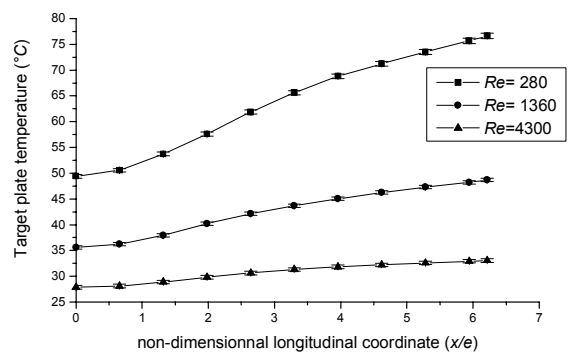

Fig. 2: Target surface temperature profiles

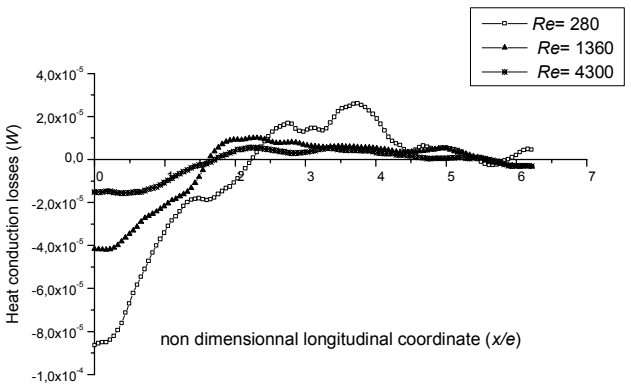

Fig. 3. Tangential conduction heat losses

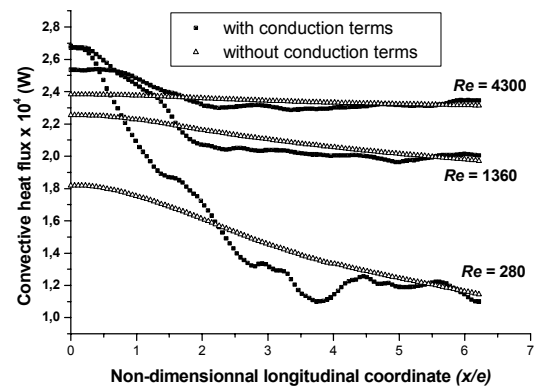

Fig. 4. Convective heat flux profiles

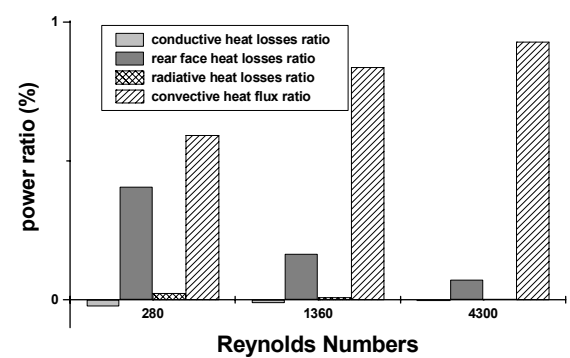

Fig. 5. Average heat fluxes along the target surface 